\title{
Electro-mechanical Behavior of 90-degree Domain Motion in Barium Titanate Single Crystals
}

\author{
Eric Burcsu, G. Ravichandran, Kaushik Bhattacharya \\ Division of Engineering and Applied Science, California Institute of Technology, \\ Pasadena, CA 91125
}

\begin{abstract}
It is well known that many common ferroelectric materials are also ferroelastic, thus the nonlinear behavior of these materials, as governed by domain motion, is highly affected by stress, as well as electric field. The combined influence of stress and electric field on domain motion and the electrostrictive response of ferroelectric single crystals is investigated. Experiments are performed on (001) and (100) oriented single crystals of barium titanate under combined electro-mechanical loading. The crystal is exposed to a constant compressive stress and an oscillating electric field along the [001] direction. Global deformation and polarization are measured as a function of electric field at different values of compressive stress. The use of semi-transparent electrodes and transmitted illumination allow in situ, real-time microscopic observations of domain motion using a long working-distance, polarizing microscope. The combined electro-mechanical loading results in a cycle of stress and electric field induced 90-degree domain switching. The magnitude of the global deformation increases with stress, with maximum steady state actuation strain of $0.57 \%$.
\end{abstract}

Keywords: ferroelectric crystals, barium titanate, domain switching, electrostriction

\section{INTRODUCTION}

Ferroelectric materials such as barium titanate have found wide application due to their excellent dielectric and electromechanical properties. Such applications include sensors and actuators for use in ultrasonics, micropositioning and active damping. The properties of interest for these applications are piezoelectricity and electrostriction. Piezoelectricity, as expressed by the converse piezoelectric effect, is a linear relation between strain and electric field, while electrostriction is a quadratic relation between strain and electric field. ${ }^{1}$ For the purposes of this paper, electrostriction will be considered in a more general sense as electric field induced deformation that is independent of field polarity. While these properties are not unique to ferroelectric materials, their behavior tends to be much more pronounced than in other materials.

Barium titanate is a ferroelectric material of the perovskite class and has been extensively studied. ${ }^{2}$ At high temperature it has the cubic structure of perovskite, as shown in figure 1 . When cooled below $120^{\circ} \mathrm{C}$, it transforms to a tetragonal phase. In addition to the strain induced by the lattice distortion, there is a spontaneous polarization along the axis of the unit cell as indicated in the figure. Thus, at phase transition the unit cell can take any of six crystallographically equivalent combinations of strain and polarization. Furthermore, different regions of a single crystal or single grain in a polycrystal can take on different directions of polarization. A region of constant polarization is known as a ferroelectric domain. Domains are separated by $90^{\circ}$ or $180^{\circ}$ domain boundaries, as shown in figure 2, which can be nucleated or moved by electric field (the ferroelectric effect) or stress (the ferroelastic effect). The process of changing the polarization direction of a domain by nucleation and growth or wall motion is known as domain switching. Electric field can induce both $90^{\circ}$ or $180^{\circ}$ switching, while stress can induce only $90^{\circ}$ switching.

Further author information:

E.B.: E-mail: burcsu@caltech.edu

G.R.: E-mail: ravi@caltech.edu

K.B.: E-mail: bhatta@caltech.edu

Smart Structures and Materials 2001: Active Materials: Behavior and Mechanics, Christopher S. Lynch,

Editor, Proceedings of SPIE Vol. 4333 (2001) @ 2001 SPIE · 0277-786X/01/\$15.00 


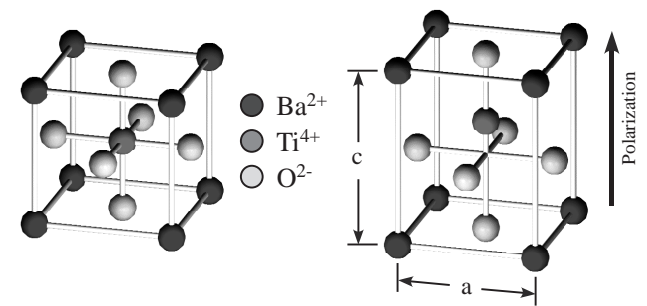

cubic

tetragonal

(b)

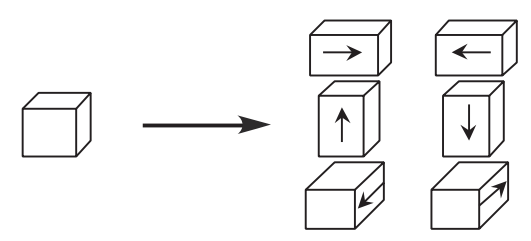

Figure 1. (a) The cubic (high temperature) and tetragonal (room temperature) structures of barium titanate. The tetragonal structure has both an induced strain and polarization. (b) Upon the cubic to tetragonal phase transition, the unit cell can take any of six equivalent combinations of strain and polarization. The arrow indicates the direction of polarization.

Domain switching is important for a number of reasons. Globally it leads to change in the macroscopic polarization vector and, in the case of $90^{\circ}$ domain switching, an associated strain that can be quite large - up to $1.1 \%$ for barium titanate and up to $6 \%$ for other materials. A ferroelectric material must have a direction of average polarization in order to be piezoelectrically active. ${ }^{3}$ However, a polycrystal, while microscopically polarized, will be macroscopically non-polar due to the random orientation of grains. Domain switching makes it possible to pole the material macroscopically by exposing it to a strong electric field. By the same token, a piezoelectric material can be depoled by electric field or stress. In addition to the poling and depoling processes, domain switching can also contribute to the enhanced piezoelectric and electrostrictive properties of some materials. ${ }^{3}$

The details of the mechanism of enhanced electromechanical properties in ferroelectrics as well as the poling and depoling process in polycrystals is very complex due to the presence of residual stresses and grain boundary mismatch. There have been a number of experimental and theoretical studies devoted to this subject for both ceramics and single crystals. ${ }^{4}$ The combined effect of stress and electric field on PLZT ceramics was studied by Lynch by measuring the nonlinear electromechanical response in presence of a uniaxial stress. ${ }^{5,6}$ Both strain and polarization were measured as a function of electric field at different stress levels. In addition, there have been many single crystal studies to understand the fundamental mechanisms governing domain switching and avoid the inherent complexity of the polycrystal system. ${ }^{7,8}$ Some recent theoretical and experimental studies have been aimed at understanding the mechanism involved in the ultra-high piezoelectric response of single crystal PMN-PT and PZNPT relaxor materials. The work of Wada, et. al. looked at the transformation strain in barium titanate in different orientations and temperatures. ${ }^{9,10}$ They measured transformation strains of $1 \%$ in $\langle 100\rangle$ oriented tetragonal barium titanate. Simulations of the underlying nature of the transformation have been performed by Fu and Cohen, though with an over prediction of the strain. ${ }^{11}$

Stress induced, $90^{\circ}$ domain switching was observed by $\mathrm{Li}$, et. al. in $\mathrm{BaTiO}_{3}$ and $\mathrm{PbTiO}_{3} .{ }^{12}$ They used a loading mechanism to generate a compressive stress along the c-axis of a crystal and observed the domain switching behavior using micro-Raman spectroscopy. For $\mathrm{BaTiO}_{3}, 90^{\circ}$ domains were shown to be nucleated by a compressive stress of $0.22 \mathrm{MPa}$. Removal of $90^{\circ}$ domains was initiated at a stress of $1.1 \mathrm{MPa}$. The current investigation focuses on the combined electromechanical response of (100) and (001) oriented barium titanate single crystals by looking at the effect of compressive stress on electric field induced domain switching and the subsequent electrostrictive response. Understanding of such a system may be useful for development of models of domain switching in grains of polycrystals. 


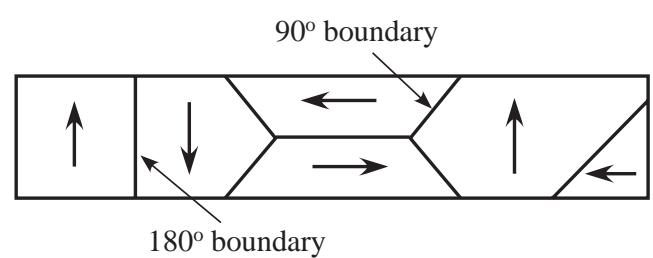

(a)

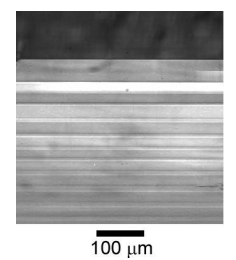

(b)

Figure 2. (a) Schematic diagram of the subgranular structure of domains, or regions of constant polarization separated by $90^{\circ}$ or $180^{\circ}$ boundaries. (b) domain pattern photographed using a polarizing microscope.

\section{THEORY}

\subsection{Energy of a Single Crystal}

A theoretical model of a ferroelectric single crystal has been developed by Shu and Bhattacharya using the framework of finite deformation continuum mechanics. ${ }^{13}$ This model is summarized below. Consider a ferroelectric single crystal occupying a region $\Omega$ in its reference (undeformed) configuration. The deformation of this crystal is $y(x)$ where $x$ is a typical material point and the polarization is $p(y)$. Note that it is natural to define the polarization in the current (deformed) configuration. Suppose this crystal is subjected to an external electric field $E_{o}$ and dead load corresponding to nominal stress $\sigma_{o}$. Then the total energy of the single crystal at temperature $\theta$ undergoing a deformation $y(x)$ and polarization $p(y)$ is given by

$$
\mathcal{E}[y, p ; \theta]=\int_{\Omega}\left\{\alpha\left|\nabla_{x} P\right|^{2}+W\left(\nabla_{x} y, P, \theta\right)-\left(\operatorname{det} \nabla_{x} y\right) E_{o} \cdot P-\sigma_{o} \cdot \nabla_{x} y\right\} d x+\frac{1}{2} \int_{\mathbb{R}^{3}}\left|\nabla_{y} \phi\right|^{2} d y
$$

where $P=p(y(x))$ and the electric potential $\phi$ is determined by solving Maxwell's equation,

$$
\nabla_{y} \cdot\left(-\nabla_{y} \phi+p\right)=\rho \quad \text { on } \mathbb{R}^{3}
$$

where $\rho$ is the free charge density and we use the convention that $p=0$ outside $y(\Omega)$.

The first term penalizes changes in the polarization, and is thus the domain wall energy. The second is the free energy density $W$ which depends on the 'deformation gradient' (or strain), the polarization and temperature. The third describes the interaction with the applied electric field and the fourth the interaction with the applied mechanical load. Finally, the second integral is the 'electrostatic' energy associated with the electric field generated by the spontaneous polarization. The equilibrium deformation and polarization can be found by minimizing the total energy over all possible deformations $y$ and polarizations $p$.

The energy density $W$ depends on the deformation gradient $F=\nabla_{x} y$ (a $3 \times 3$ matrix), the polarization $P$ (a vector) and temperature $\theta$. It is frame-indifferent, i.e.,

$$
W(Q F, Q P, \theta)=W(F, P, \theta) \text { for all rotation matrices } Q
$$

satisfies material symmetry and has ground states (minima) corresponding to the observed spontaneously polarized state of the crystal. For barium titanate, in the absence of any field or load, material symmetry implies that at room temperature it can be polarized in any of the six $\langle 100\rangle$ directions with its associated deformation. These are the crystallographically equivalent structures mentioned earlier. Thus there are six symmetry related ground states for which $W$ is minimized. Minimization of the total energy dictates that external mechanical or electrical work can result in an exchange of stability between different ground states.

\subsection{Flat Plate Configuration}

For a flat plate configuration with electrodes on each face, the minimization problem can be simplified significantly. If the plate is thin and is bounded by electrodes on the two faces, the last integral in equation (1) can be neglected. 


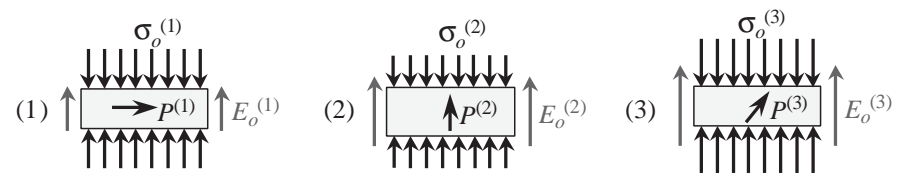

Figure 3. Possible flat plate equilibrium configurations under compressive stress and electric field loading.

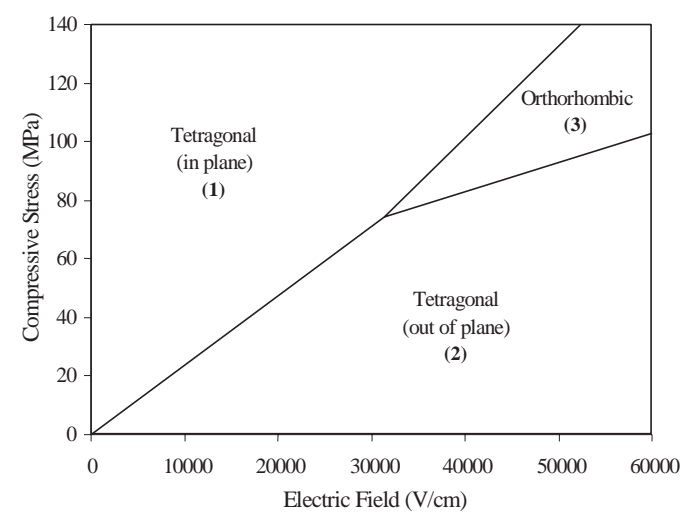

Triple Point: $\sigma_{o}=-74 \mathrm{MPa}, E_{o}=31,000 \mathrm{~V} / \mathrm{cm}$

Figure 4. Compressive stress-electric field phase diagram of barium titanate at room temperature.

In addition, if the size of the crystal is much larger than the domain wall, the domain wall energy can be neglected. The problem can then be reduced to the minimization of the following energy density function:

$$
G(F, P, \theta)=W(F, P, \theta)-(\operatorname{det} F) E_{o} \cdot P-\sigma_{o} \cdot F
$$

The possible equilibrium configurations for a crystal of barium titanate cut in a flat plate configuration with a (100) orientation under combined electromechanical loading, are shown in figure 3. Any configuration with the polarization oriented in-plane will be energetically equivalent. This is shown as state (1), with some combination of stress and electric field for which this state is stable. At some different combination of compressive stress and electric field, the stable equilibrium configuration will be state (2), the out-of-plane configuration. It also happens that for very high values of compressive stress and electric field, the orthorhombic phase is the equilibrium configuration, shown as state (3). Minimization of equation (4) results in a phase diagram outlining the equilibrium boundaries of the three configurations. The phase diagram for barium titanate at room temperature is shown in figure 4 . It is important to note that the configurations mentioned are equilibrium states. The problem of switching between equilibrium states is kinetic in nature.

\subsection{Mode of Electrostrictive Actuation}

The exchange of stability between the in-plane and out-of-plane configurations discussed in the previous section suggests a potential mode of operation for an electromechanical actuator. This mode of operation takes advantage of the change in strain associated with switching between states (1) and (2) shown in figure 3 . A single crystal ferroelectric in a flat plate configuration, with (100) orientation is subjected to a constant, uniaxial compressive prestress with no electric field. The equilibrium configuration is thus state (1). A voltage is introduced of sufficient magnitude to switch the crystal to state (2). The voltage is subsequently removed and compressive stress causes the crystal to return to state (1). The combined electromechanical loading allows a cyclic change in the domain pattern resulting in an electrostrictive strain limited by the c/a ratio of the given crystal. For barium titanate this corresponds to a strain of $1.1 \%$. Other materials could produce higher strains, as large as $6 \%$. 

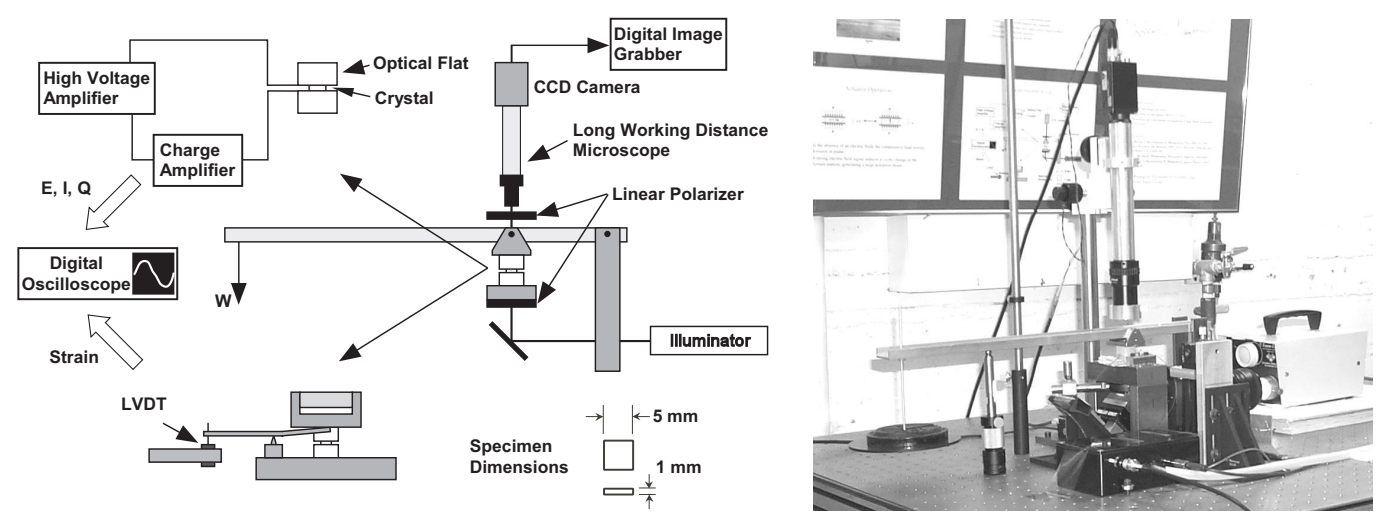

Figure 5. Experimental setup and specimen dimensions.

\section{EXPERIMENT}

An experimental setup was designed to demonstrate the principles outlined above. The system was designed to apply a constant compressive load and variable electric field to a ferroelectric crystal. It was further designed to allow accurate measurement of strain and polarization, as well as allow in situ observation of the crystal during the experiment. Experiments were performed on single crystals of barium titanate of 5x5x1mm dimensions and (001) or (100) orientation (Superconix, Lake Elmo, MN). The crystals were poled prior to polishing, so were nearly single domain in most cases. Barium titanate was chosen because it has been studied extensively and can be obtained in single crystal form.

The experimental setup consists of a loading mechanism, displacement measurement transducer, high-voltage power supply and long working distance video microscope. A schematic diagram and photograph of the system are shown in figure 5 . The loading mechanism uses dead weight $W$ and a lever to deliver a force to a loading frame. The force is transmitted to a pair of optically flat glass plates that sandwich the specimen. Glass plates are used so that the entire load axis is transparent, allowing direct observation of the specimen during the test. Semi-transparent, platinum electrodes are deposited on the surface of the crystal. Platinum lines are also deposited on the surface of the glass plates. Small dots of conductive carbon grease are applied to the corners of each surface of the crystal to ensure good electrical contact between the electrodes and the platinum lines and allow some degree of lubrication. Wires are bonded to the lines using conductive epoxy (Chemtronics CW2400).

The electrical input is generated using a function generator and a high voltage power amplifier (Trek, Inc. Model 10/10B). Experiments are carried out in the static or pseudo-static regime with input signal frequencies between 0.05 Hz. and $1 \mathrm{~Hz}$. Electric field is measured by monitoring the amplifier voltage. Crystal strain is measured by recording the load frame displacement using a high-resolution LVDT (Lucas-Schaevitz 025-MHR). A lever is actuated at the center of the loading frame causing a displacement of the LVDT and providing a mechanical amplification. The displacement corresponds to the change in thickness of the specimen. In addition, charge on the crystal is measured using an integrating capacitor. The voltage, current, charge and displacement signals are recorded using a digital oscilloscope.

Real time, in situ observations are made of the crystal using a long working distance video microscope. The microscope consists of a 10x long working distance objective (Nikon), extension tube and CCD video camera (Sony $\mathrm{XC}-75)$ connected to a digital frame grabber (Epix SV-4). This system has a field of view of about $500 \mu \mathrm{m}$ and lateral resolution of approximately $1 \mu \mathrm{m}$ at a working distance of $50 \mathrm{~mm}$. Light is transmitted from a high intensity halogen lamp through crossed polarizers, one below the specimen and one above. Images are captured at up to 30 frames per second, allowing observation of domain switching and failure that occurs during the course of the experiment. 


\section{RESULTS}

Experiments have been performed on crystals to investigate the stress dependence of the electrostrictive strain response. During the experiment, a slow voltage signal is generated and the strain and polarization are measured. Figure 6 shows data from a typical experiment with an input signal frequency of $0.05 \mathrm{~Hz}$. The electric field input signal is shown as the solid curve in both plots. The dashed curves in figure 6(a) and 6(b) are the measured strain and polarization signals, respectively. As the electric field increases, there is a sudden increase in both strain and polarization, followed by a leveling off. As the electric field decreases, the strain drops and then increases again for negative electric field, while the polarization continues to decrease before leveling off again. Because of the symmetric behavior of the strain with respect to polarity of the electric field, the deformation can be considered to be electrostrictive in the general sense mentioned in section 1.

A series of strain-electric field trajectories are shown in figure 7 at five values of compressive stress. The data shown are from the fifth cycle of each experiment for an initially (100) oriented crystal. The first plot has a compressive stress of approximately zero (there is a small stress present due to the measurement method). In this case, the total strain is less than $0.1 \%$. With subsequent increase in compressive stress, there is an increase in the maximum strain up to $0.57 \%$ at $2.14 \mathrm{MPa}$, and a broadening of the butterfly hysteresis loops. Figure 8 shows a series of polarizationelectric field trajectories for the same cases. For the zero stress case, the hysteresis curve has very sharp corners as is usually observed for single crystal ferroelectrics. As the stress is increased, there is a blunting of the corners.

The actuation strain as a function of compressive stress is summarized in figure $9(\mathrm{a})$. The actuation strain is defined as the difference between the maximum and minimum strain for a given half cycle and was calculated in the fifth cycle of each experiment. Data for initially (100) and (001) oriented crystals are shown. There is a clear increase in actuation strain with increasing stress in each case. The (100) crystal seems to have a greater stress sensitivity than the (001) crystal, this may be due to variations in the quality of the material or a preference for the original structure due to residual stresses or the presence of defects. Coercive field, defined as the field required to reduce the polarization to zero, is shown as a function of compressive stress in figure 9 (b). The coercive field is found to be relatively insensitive to stress with an increase of about 20-30 V/cm/MPa.

Frequency dependence of the strain response at different stresses is also of interest. The experimental setup is only suitable for static, or very low frequency experiments, however, limited investigation is possible. Figure 10 shows the strain-electric field trajectories for input signal frequencies of $0.05 \mathrm{~Hz} ., 0.1 \mathrm{~Hz}$. and $1.0 \mathrm{~Hz}$. In each case, the crystal was loaded at $1.07 \mathrm{MPa}$ compressive stress. The maximum actuation strain for the tests increases with increasing input frequency.

Observations are made of the crystal domain patterns during the experiment. Figure 11 shows two images of domain patterns. These images are chosen from a series of images taken over the course of one cycle on an initially (001) oriented crystal at a compressive stress of 3.2 MPa. The first image (a), is taken at an electric field of $30 \mathrm{~V} / \mathrm{cm}$. The second image (b), is taken at a field of $1400 \mathrm{~V} / \mathrm{cm}$. On the left side of the image, horizontal domain boundaries are visible with a vertical band on the right and very narrow domains, or needles, terminating at $45^{\circ}$. At $1400 \mathrm{~V} / \mathrm{cm}$, several of these needles have disappeared. During the experiment, a series of pictures are taken so that the evolution of this microstructure can be observed. Cracks are found to form in the crystals during the experiments. In some cases, after several sets of experiments, the crystal is broken into a number of pieces. In general, however, the (100) crystals tend to form more cracks than the (001).

\section{DISCUSSION}

The experimental data show the variation of steady-state actuation strain in (100) and (001) oriented crystals of up to about $0.57 \%$ in a range of stress of 0 to $3.2 \mathrm{MPa}$, about half of the $1.1 \%$ theoretically predicted maximum strain. Evidence from the in situ microscopy and polarization data, indicate that nearly complete out of plane polarization is reached at the high voltage state, however, the strain data indicates that the complete in-plane state is not reached. Factors such as presence of defects and friction between the crystal and the loading surfaces may prevent complete transition to the in-plane state. Experiments at higher stresses are needed to determine the practical limits on the actuation strain. 


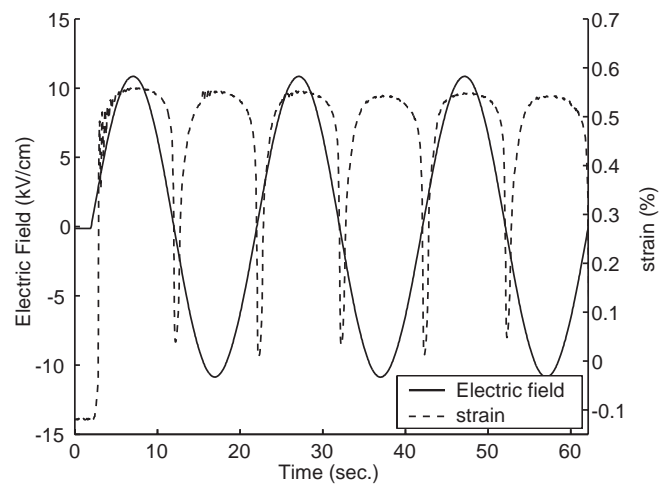

(a)

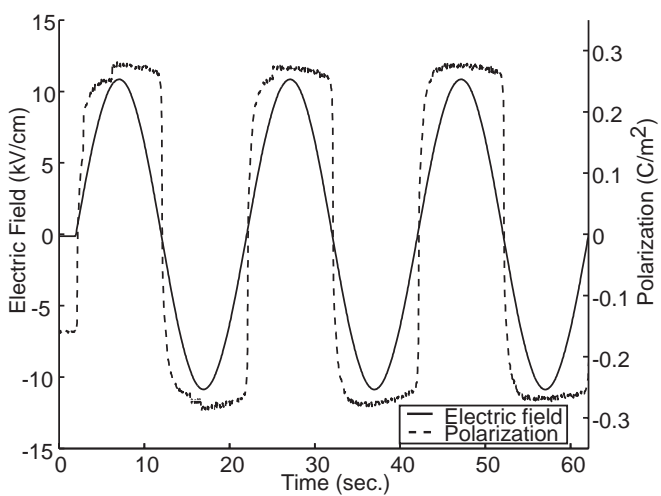

(b)

Figure 6. (a) Strain vs. time and (b) polarization vs. time for an initially (100) oriented crystal at a compressive stress of $1.07 \mathrm{MPa}$.
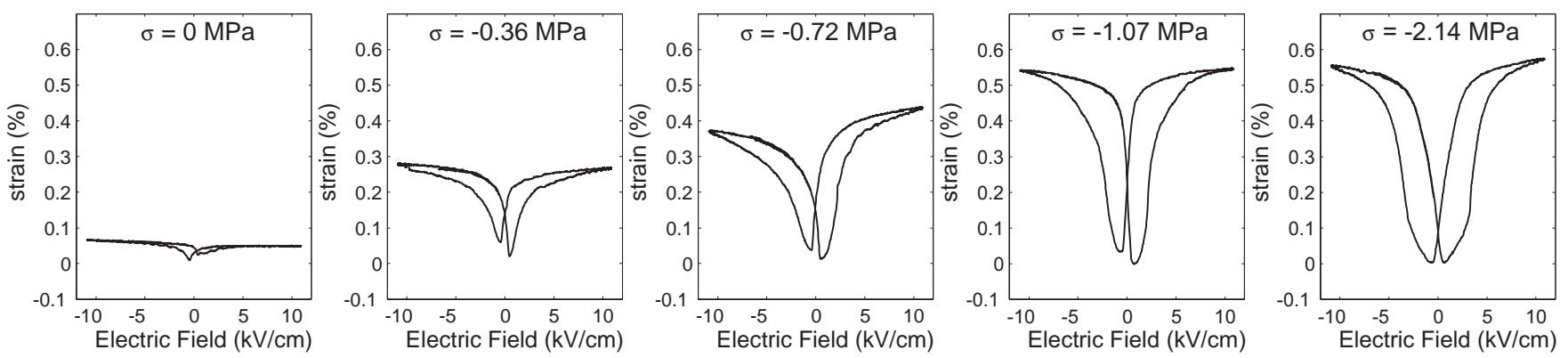

Figure 7. Strain vs. electric field for an initially (100) oriented crystal at five values of compressive stress.
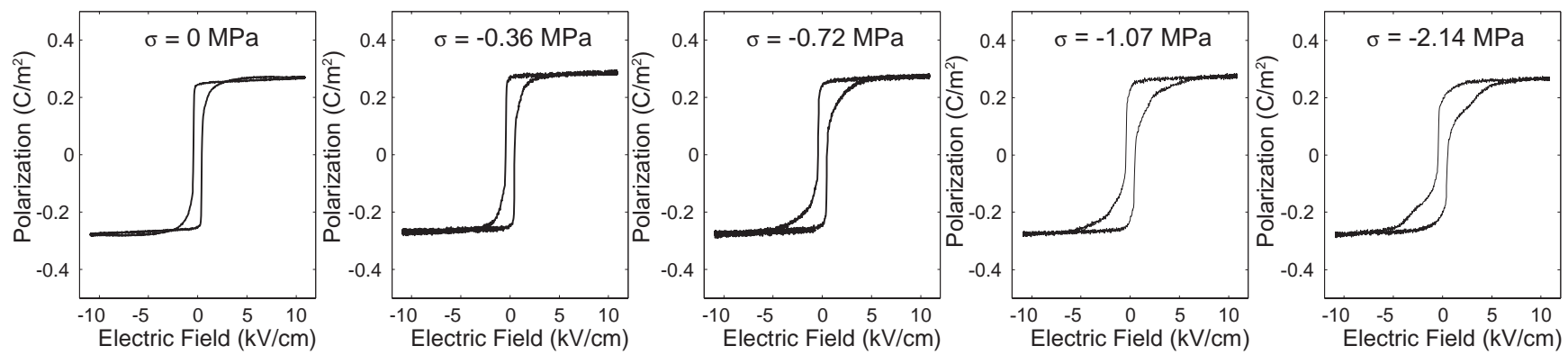

Figure 8. Polarization vs. electric field for an initially(100) oriented crystal at five values of compressive stress. 


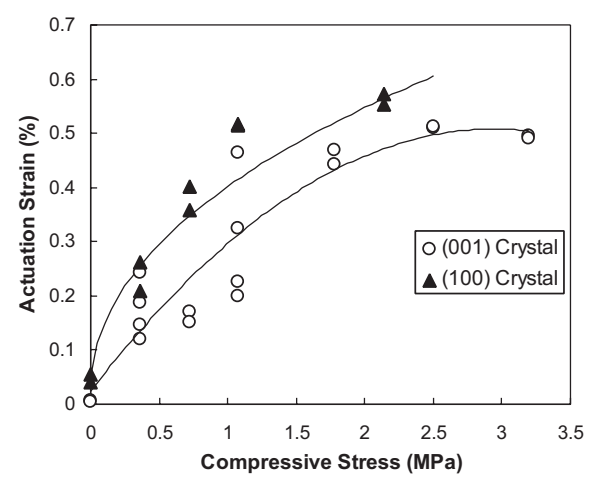

(a)

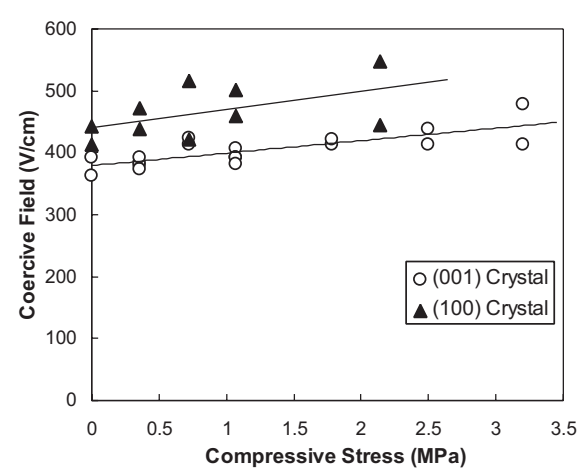

(b)

Figure 9. (a) Actuation strain and (b) coercive field during fifth cycle of test as a function of compressive stress for initially (100) and (001) oriented crystals.

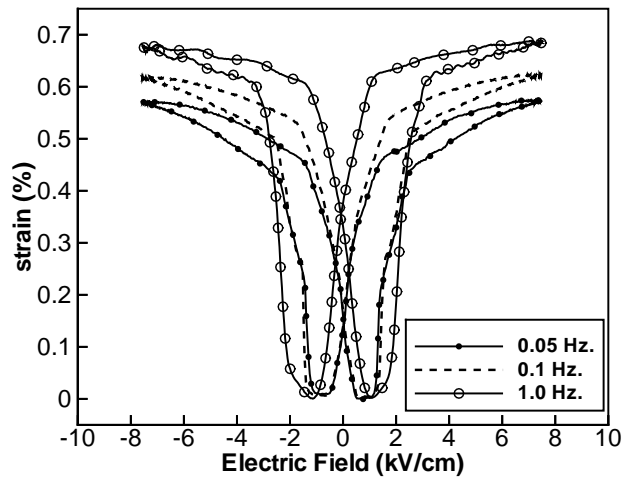

Figure 10. Strain vs. electric field at different frequency for an initially (001) oriented crystal at $1.07 \mathrm{MPa}$ compressive stress.

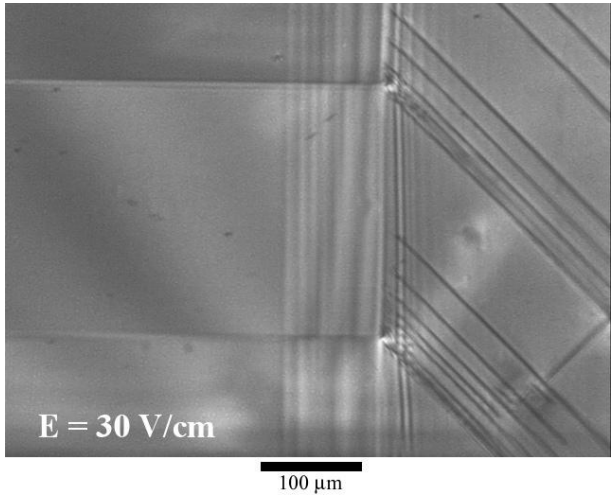

(a)

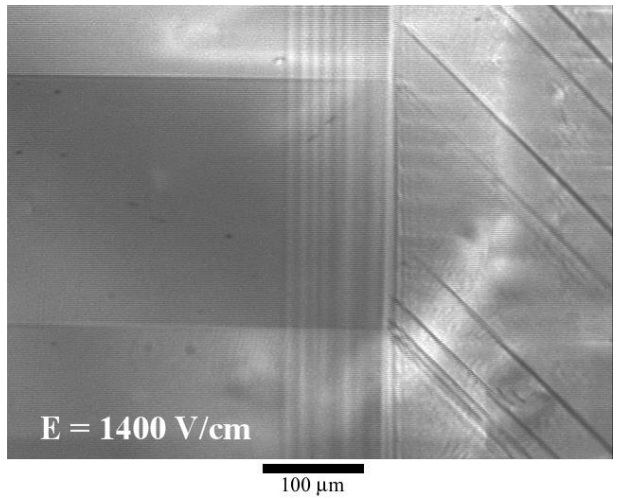

(b)

Figure 11. Images of domain pattern in initially (001) oriented crystal under $3.2 \mathrm{MPa}$ compressive stress at an electric field of (a) $30 \mathrm{~V} / \mathrm{cm}$ (b) $1400 \mathrm{~V} / \mathrm{cm}$. 
While the theory deals with equilibrium states, the kinetics dictate the path between states. Even at these low frequencies, there may not be enough time to settle into a completely in-plane equilibrium state. This idea motivated the limited investigation of frequency response. The data, however, indicate that the maximum actuation strain increases with input frequency. The reasons behind this behavior are unclear and require further study. In the future, an improved apparatus will be used to explore a greater range of the frequency response.

Cracks are found to form in the crystals during the experiments. This is not surprising considering the large strains involved. In general, however, the (100) crystals tend to form more cracks than the (001). This is somewhat surprising since after several cycles, one may assume that the original state will have little or no effect on the current behavior. An explanation may be simply differences in quality of the crystals or density of preexisting microcracks or flaws. It may be that most cracks or microcracks form during the initial switching cycle for the (100) crystals which experience a 100\% (100) to (001) transformation. Crack growth will then continue over the remainder of the experiment. The (001) crystals, on the other hand, never go through a complete $90^{\circ}$ transition at the levels of stress used.

\section{CONCLUDING REMARKS}

An experimental setup has been designed to investigate the stress dependence of the electrostrictive strain response of ferroelectric single crystals. Experiments have been performed on initially single domain crystals of barium titanate $\left(\mathrm{BaTiO}_{3}\right)$ with (100) and (001) orientation at compressive stresses between 0 and $3.2 \mathrm{MPa}$. A very clear increase in steady state actuation strain is seen with increased compressive stress with maximum strains of around $0.57 \%$. A very limited investigation of the frequency dependence of the strain response has been started, however much more work needs to be done in this area. Initial data indicates an increase in actuation strain as frequency is increased in the low frequency regime. An important question to address is what levels of actuation strain are possible at higher frequencies where one might attempt to apply this method of actuation to do work for microswitches or active structures. Future work on barium titanate will focus on this as well as the variation with temperature. Similar experiments will be carried out on bulk crystals of lead titanate and thin film materials.

\section{ACKNOWLEDGMENTS}

We gratefully acknowledge the support of the Army Research Office (M.A. Zikry, Program Manager) under grant DAAD 19-99-1-0319. This work was supported in part by the MRSEC Program of the National Science Foundation under Award Number DMR-0080065.

\section{REFERENCES}

1. K. Uchino, Ferroelectric Devices, Marcel Decker, New York, 2000.

2. F. Jona and G. Shirane, Ferroelectric Crystals, Dover, New York, 1993.

3. L. E. Cross, "Ferroelectric materials for electromechanical transducer applications," Jpn. J. Appl. Phys. Pt. 1 34, pp. 2525-2532, 1995.

4. S. P. Li, A. S. Bhalla, R. E. Newnham, L. E. Cross, and C. Y. Huang, " $90^{\circ}$ domain reversal in $\mathrm{Pb}\left(\mathrm{Zr}_{x} \operatorname{Ti}_{1-x}\right) \mathrm{O}_{3}$ ceramics," J. Mater. Sci. 29, pp. 1290-1294, 1994.

5. C. S. Lynch, "The effect of uniaxial stress on the electro-mechanical response of 8/65/35 PLZT," Acta mater. 44, pp. 4137-4148, 1996.

6. W. Chen and C. S. Lynch, "A micro-electro-mechanical model for polarization switching of ferroelectric materials," Acta mater. 46, pp. 5303-5311, 1998.

7. R. C. Miller and A. Savage, "Motion of $180^{\circ}$ domain walls in metal electroded barium titanate crystals as a function of electric field and sample thickness," J. Appl. Phys. 31, pp. 662-669, 1960.

8. E. A. Little, "Dynamic behavior of domain walls in barium titanate," Phys. Review 98, pp. 978-984, 1955.

9. S. Wada, S. Suzuki, T. Noma, T. Suzuki, M. Osada., M. Kakihana, S. E. Park, L. E.Cross, and T. R. Shrout, "Enhanced piezoelectric property of barium titanate single crystals with engineered domain configurations," Jpn. J. Appl. Phys. 38, pp. 5505-5511, 1999. 
10. S. E. Park, S. Wada, L. E. Cross, and T. R. Shrout, "Crystallographically engineered $\mathrm{BaTiO}_{3}$ single crystals for high-performance piezoelectrics," J. Appl. Phys. 86, pp. 2746-2750, 1999.

11. H. Fu and R. E. Cohen, "Polarization rotation mechanism for ultrahigh electromechanical response in singlecrystal piezoelectrics," Nature 403, pp. 281-283, 2000.

12. Z. Li, C. M. Foster, X.-Z. Dai, X.-Z. Xu, S.-K. Chan, and D. J. Lam, "Piezoelectrically-induced switching of $90^{\circ}$ domains in tetragonal $\mathrm{BaTiO}_{3}$ and $\mathrm{PbTiO}_{3}$ investigated by micro-Raman spectroscopy," J. Appl. Phys. 71, pp. 4481-4486, 1992.

13. Y. C. Shu and K. Bhattacharya, "Domain patterns and macroscopic behavior of ferroelectric materials," Phil. Mag. A (submitted). 